\title{
Prevalence of HBV and HCV Infections in Iranian Blood Donors; An Updated Systematic Review and Meta-Analysis
}

\author{
Leila Kasraian ${ }^{1}$, Mohammad Hossein Imanieh ${ }^{2, *}$, Reza Tabrizi ${ }^{3,4}$, Reza Shahriarirad ${ }^{5}$, \\ Amirhossein Erfani ${ }^{5}$, Sahar Hosseini ${ }^{5}$
}

1. Department of Fars Blood Transfusion Research Center, Shiraz University of Medical Sciences, Shiraz, Iran

2. Gastroenterohepatology Research Center, Shiraz University of Medical Sciences, Shiraz, Iran

3. Non-Communicable Diseases Research Center, Fasa University of Medical Sciences, Fasa, Iran

4. Health Policy Research Center, Institute of Health, Shiraz University of Medical Sciences, Shiraz, Iran

5. Student Research Committee, Shiraz University of Medical Sciences, Shiraz, Iran

\footnotetext{
* Corresponding Author:

Mohammad Hossein Imanieh, MD Gastroenterohepatology Research Center,Nemazee Hospital Nemazee square, Shiraz University of Medical Sciences, Shiraz, Iran

Telefax: + 987136474316

Email: imaniehmh@sums.ac.ir

Received: 16 Dec. 2020

Accepted: 22 May 2021
}

\section{ABSTRACT}

\section{BACKGROUND}

Awareness of the prevalence of hepatitis $\mathrm{B}(\mathrm{HBV})$ and hepatitis $\mathrm{C}$ virus (HCV) infections, as blood transmitted infections, among blood donors can help policymakers improve the guidelines, share experiences, and estimate the blood safety over the country and in the region. We aimed to determine the prevalence of HBV and HCV infection in Iranian blood donors based on the present published literature.

\section{METHODS}

A meta-analysis was carried out based on the results of an electronic literature search in the international and national databases for all articles published until October 2020. We selected studies that had appropriate sampling and valid statistical analysis as well as proper measurement methods. The heterogenic indices of the studies were determined using Cochran's (Q) and I-square (I2) tests. According to the heterogeneity results, a fixed or random-effects model was implemented to estimate the pooled prevalence of HBV and HCV. Meta-regression was conducted to explore the suspected sources of heterogeneity.

\section{RESULTS}

We included 61 and 58 eligible studies related to $\mathrm{HBV}$ and $\mathrm{HCV}$, respectively. The pooled prevalence of HBV was $0.57 \%$ (95\% confidence interval $(\mathrm{CI}): 0.47-0.67, \mathrm{I} 2$ : 99.9\%) among the blood donors. The range of prevalence rates of HBV was between $0.10 \%$ and $2.34 \%$ in different areas of Iran. The pooled prevalence of HCV was $0.22 \%$ (95\% CI: $0.20-0.24$, I2: $98.64 \%$ ) in blood donors, which varied between $0.02 \%$ and $1.09 \%$ in separate locations. Subgroup and meta-regression analyses revealed that the year of publication, geographical location, and quality of the studies probably generated the heterogeneity.

\section{CONCLUSION}

The prevalence of HBV and HCV decreased steadily in Iranian blood donors during the past two decades. It should be asserted that most of the health policies and safety measures taken in recent years in Iran have been effective and promising.

\section{KEYWORDS:}

Hepatitis B, Hepatitis C, Prevalence, Blood donation, Blood donor

Please cite this paper as:

Kasraian L, Imanieh MH, Tabrizi R, Shahriarirad R, Erfani A, Hosseini S. Prevalence of HBV and HCV Infections in Iranian Blood Donors; An Updated Systematic Review and Meta-Analysis. Middle East J Dig Dis 2021;13:236-251. doi: 10.34172/mejdd.2021.231. 


\section{INTRODUCTION}

Estimations report that 257 million and 71 million people suffer from chronic hepatitis $\mathrm{B}$ (HBV) and $\mathrm{C}$ viruses (HCV), respectively. About 1.34 million deaths were attributed to chronic infection with $\mathrm{HBV}$ and $\mathrm{HCV}$ in 2015 worldwide, most of which was due to complications of long-standing hepatitis such as liver cirrhosis and hepatocellular carcinoma (HCC). ${ }^{1,2}$ Both viruses can be transmitted through contact with infected blood or blood products. While the world health organization (WHO) recommended screening of all blood donations for evidence of infections such as viral hepatitis, blood safety has been determined as a major public health priority since 2000 . $^{2,3}$ Careful selection of people who tend to donate blood became a crucial part of the management of blood safety, and the strategy of recruiting voluntary non-remunerated donors (VNRDs) and encouraging them to become regular donors has led to an improvement in blood safety. ${ }^{4,5}$

In Iran, the screening of blood donors for hepatitis B surface antigen (HBsAg) has become mandatory since the foundation of the Iranian Blood Transfusion Organization (IBTO) in $1974 .{ }^{6}$ As a national public health promotion program started in 1993, vaccination of neonates against $\mathrm{HBV}$ infection has changed the epidemiology of the infection in the country. ${ }^{7}$ The estimated prevalence of HBsAg in the general population of Iran is $2.2 \%$, and the infection is more common among men than women ( $3 \%$ vs. $1.7 \%$ ). ${ }^{8}$ In Iran, the government pays the costs of collection, preparation, preservation, and distribution of blood and its products. VNRDs provide $100 \%$ of blood donations; hence, patients receive the blood products free of charge. ${ }^{9}$ According to the literature, the pooled prevalence of HBsAg in blood donors of Iran was estimated to be $0.58 \%$. $^{3}$

$\mathrm{HCV}$ infection is often asymptomatic or has mild symptoms during the acute phase of infection. The infection becomes chronic in most cases and is a major risk factor for developing cirrhosis and HCC. ${ }^{10}$ Viral variability and great adaption ability are certain challenges for $\mathrm{HCV}$ vaccine development. ${ }^{11}$ Before the early 1990 s, the main risk factors for transmission of $\mathrm{HCV}$ infection were blood transfusion, intravenous drug use, and unsafe injection procedures. ${ }^{10}$ In Iran, screening of all blood donors for anti$\mathrm{HCV}$ antibody (Ab) has been performed since $1996 .^{12}$
This has changed the principal route of $\mathrm{HCV}$ transmission from blood transfusion to intravenous drug use, and by considering the growth in the number of people who inject drugs, the prevalence of $\mathrm{HCV}$ infection is increasing in the country. ${ }^{13}$ Previous reports estimated the prevalence of anti-HCV Ab in the general population of Iran to be approximately $0.6 \% .{ }^{14}$ The pooled prevalence of anti$\mathrm{HCV}$ in blood donors was also $0.5 \%{ }^{15}$

The objective of the present study was to review the articles on the prevalence of HBV and HCV infections in Iranian blood donors to make an updated estimation of the burden of these infections in this population.

\section{MATERIALS AND METHODS}

\section{Search Strategy}

The current meta-analysis was performed according to the criteria of the PRISMA guidelines. ${ }^{16}$ An electronic systematic search algorithm in the international databases, including PubMed, Scopus, Embase, and Google scholar, was adopted for articles published until October 2020 using the following keywords: ("blood donor" OR "blood donation" OR "bloodborne pathogens" OR "blood transfusion" OR "transfusion-transmitted infection") AND ("prevalence" OR "epidemiology") in combination with ("Iran") and also ("hepatitis B" OR "HBV" OR "hepatitis C" OR "HCV") for hepatitis B and hepatitis C as keywords for titles and/or abstracts in a medical subject headings (MeSH) word search. The Google scholar and Iranian databases, including Scientific Information Database (SID) and Magiran were searched for published articles in the Persian language with Persian equivalents of the aforementioned keywords. References of the reviews, systematic reviews and meta-analyses, and relevant retrieved articles were searched to increase the sensitivity.

\section{Eligibility Criteria and Study Selection}

Studies that recruited Iranian blood donors, published in Persian or English languages, were used for the current review article. All included studies must measure and report the prevalence of individuals with positive HBsAg and/ or anti-HCV tests. The seropositive results must be confirmed by HBsAg confirmatory assay and recombinant immunoblot assay (RIBA) test for HBV and HCV diagnosis, respectively. The exclusion criteria were (1) no accessible 
full text or insufficient statistical information about the prevalence and the number of the infected cases; (2) studies performed exclusively on the specific blood donors' population (i.e., recruitment of only $\mathrm{HBV} / \mathrm{HCV}$ positive blood donors).

\section{Data Collection and Quality Assessment}

One reviewer (LK) extracted the data and doublechecked for the following items: authors' names, publication year, study period, location, sample size, the prevalence of HCV and HBV, and blood donation status (first time, lapsed, and regular blood donor). In case there was ambiguity about the information extraction, the problem was resolved by the other author as well (MHI).

After determining the relevant articles, two of the authors ( $\mathrm{SH}$ and $\mathrm{MHI}$ ) assessed the quality of the studies by an adapted version of the Newcastle-Ottawa Quality Scale (NOS) for cross-sectional studies. ${ }^{17}$ This scale consists of seven items in three distinct categories, including selection, comparability, and outcome. A star scoring system is predicted for each item to provide a semi-quantitative evaluation of the study quality ranging from 0 to 9 . Studies with $<7$ stars were defined as low quality and 7 or more than 7 stars as high quality.

\section{Statistical analysis}

Meta-analysis was conducted using Metaprop command in STATA version 14.0 (Stata Corp., College Station, TX, USA).${ }^{18}$ Due to the closeness of the prevalence of HBV and HCV to zero, Freeman-Tukey double arcsine transformation was employed; also, a 95\% confidence interval was computed by using the exact binomial method. Cochran Q test and I-square (I2) were employed to assess the heterogeneity. Whenever heterogeneity of the study was significant (Cochran Q $p$-value $<0.1$ and $\mathrm{I} 2 \geq 50 \%$ ), a random effect meta-analysis was used; otherwise, a fixed effect meta-analysis was applied to combine the prevalence. Meta-regression analyses were conducted for continuous variables, including the date of publication and total sample size among the studies to explore the source of heterogeneity. Besides, we performed sensitivity analyses using leave-one-out to examine the effects of one by one included studies on the stability of the pooled effect sizes.

\section{RESULTS}

In the electronic search in PubMed, Scopus, Embase, and other sources, 144, 224, 427, and 7743 articles were identified, respectively. After narrowing down the search strategy and removing repetitive material due to overlapping contents of the databases, 2968 documents remained. In the next step and thorough screening of the titles and abstracts, duplicated studies (1675) and irrelevant ones (1216) were excluded. Ultimately, the remaining 77 full-text articles were examined, and after omitting 12 papers, 65 articles ${ }^{6,19-82}$ met the inclusion criteria, including 61 studies for hepatitis B and 58 studies for hepatitis $\mathrm{C}(7,4$, and 54 studies were reporting $\mathrm{HBV}, \mathrm{HCV}$, and the prevalence of both viruses, respectively). Only crosssectional and case-control studies were selected for final analysis, and case reports, case series, and letters were omitted. The step-by-step study identification and selection process based on literature findings are presented in figure 1 . The basic characteristics of the included studies are summarized in table 1 .

\section{Hepatitis B}

The total sample size that were included in the meta-analysis was $27,672,938$ ranging from $441^{43}$ to

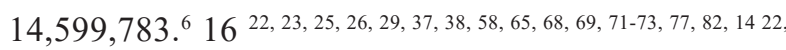
$23,25,26,29,37,38,54,58,69,71-73,77$, and $11^{22,23,26,29,37,38,58,69,71,72,77}$ studies had reported the prevalence of HBV among the first time $(1,877,630$ donors ranging from 2,664 to $1,137,582)$, lapsed $(1,719,389$ donors ranging from 2,864 to 914,026$)$, and regular $(1,681,469$ donors ranging from 728 to $1,002,984$ ) blood donors, respectively.

15 studies 22-27,29,31,35,39,45,69,73,76,81 had provided the number of HBV cases $(15,733 \mathrm{HBV}$ cases ranging from 11 to 9,944) among male donors $(5,035,693$ male donors ranging from 2,787 to 2,240,419); and, 15 studies $22-27,29,31,35,39,45,69,73,76,81$ had provided number of HBV cases (1,121 HBV cases ranged from 0 to 704$)$ among female donors (332,793 female donors ranging from 213 to 172,856).

Using a random-effects model based on 61 studies, the pooled prevalence of HBV was $0.57 \%$ (95\% CI: $0.47-$ 0.67 ) among blood donors (figure $2 \mathrm{~A}$ ). According to the significant heterogeneity (I2: $99.9 \%, p<0.001$ ), additional analyses were conducted. Meta-regression analysis results showed that factors such as the date of publication $(\beta=-0.05$, 


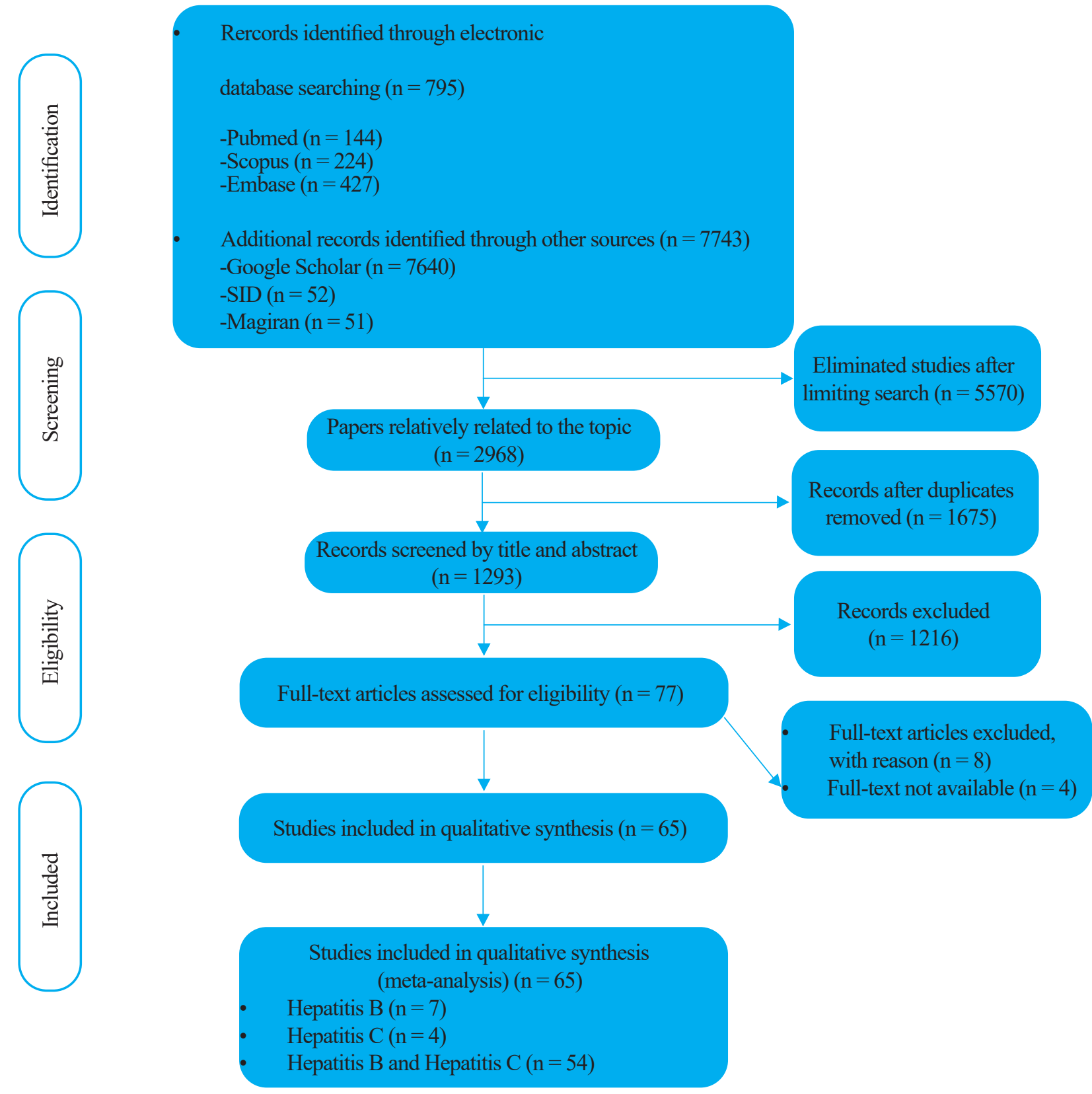

Fig.1: The flowchart of the identification of the studies and selection process

$p<0.001$ ) were associated with HBV prevalence (figure $3 \mathrm{~A})$, but there was no significant impact on the total sample size $(\beta=0.00, p=0.671)$.

Based on the sensitivity analysis, we found that the highest pooled prevalence of HBV was $0.58 \%$ (95\% CI: 0.48- 0.68) after excluding the study by Etminan and colleagues, 38 and the lowest pooled prevalence of $\mathrm{HBV}$ was $0.54 \%$ (95\% CI: $0.44-0.64)$ after excluding the article by Sanei Moghaddam and co-workers. ${ }^{73}$ The pooled preva- lence of HBV based on blood donors' conditions, sex, and quality status is shown in table 2.

\section{Hepatitis C}

The total sample size included in the meta-analysis was $12,686,297$ ranging from $441^{43}$ to $2,413,275 .{ }^{23} 14{ }^{23,25,}$ $26,29,37,38,58,65,68,71-73,77,82,12^{23,25,26,29,37,38,54,58,71-73,77}$, and 9 $23,26,29,37,38,58,71,72,77$ studies had reported the prevalence of $\mathrm{HCV}$ among the first time $(1,852,977$ donors ranging from 
Table 1: The basic characteristics of the included studies

\begin{tabular}{|c|c|c|c|c|c|c|}
\hline \multirow{2}{*}{ Authors } & \multirow{2}{*}{ Year } & \multirow{2}{*}{ Province } & \multicolumn{2}{|c|}{ For HBV } & \multicolumn{2}{|c|}{ For HCV } \\
\hline & & & No. HBV & Total & No. HCV & Total \\
\hline Afzali et al ${ }^{19}$ & 2002 & Esfahan & 273 & 44004 & 477 & 43731 \\
\hline Aghajanipoor et al ${ }^{20}$ & 2006 & Ardebil & 213 & 16789 & 79 & 16576 \\
\hline Aghamohammadi et al ${ }^{21}$ & 2014 & Mazandaran & 297 & 125001 & 32 & 124704 \\
\hline Alaei et $\mathrm{al}^{22}$ & 2019 & Mazandaran & 174 & 132124 & & \\
\hline Amini Kafi-abad et al \#1 ${ }^{6}$ & 2009 & Iran & 140367 & 14740150 & & \\
\hline Amini Kafi-abad et al \#2 23 & 2009 & Iran & 10648 & 2423923 & 3146 & 2413275 \\
\hline Arab et $\mathrm{al}^{24}$ & 2006 & Kerman & 162 & 15535 & & \\
\hline Attarchi et al ${ }^{25}$ & 2006 & Tehran & 166 & 26811 & 42 & 26645 \\
\hline Azadbakht et al ${ }^{26}$ & 2020 & Fars & 2684 & 1955162 & 1703 & 1952478 \\
\hline Bani Aghil et al ${ }^{27}$ & 2010 & Golestan & 1271 & 129469 & 161 & 128198 \\
\hline Boustani et $\mathrm{al}^{28}$ & 2017 & Ilam & 102 & 72629 & 27 & 72527 \\
\hline Bozorgi et al ${ }^{30}$ & 2006 & Qazvin & 218 & 48334 & 73 & 48116 \\
\hline Bozorgi et al $^{29}$ & 2012 & Qazvin & 47 & 20638 & 35 & 20591 \\
\hline Dargahi et $\mathrm{al}^{31}$ & 2012 & Ardebil & 94 & 26595 & 6 & 26501 \\
\hline Delavari et al ${ }^{32}$ & 2005 & Kerman & & & 59 & 15252 \\
\hline Doosti et al ${ }^{33}$ & 2009 & $\begin{array}{l}\text { Chaharmahal } \\
\text { \& Bakhtiari }\end{array}$ & 200 & 11400 & 76 & 11200 \\
\hline 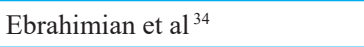 & 2011 & Esfahan & 1066 & 543771 & 670 & 542705 \\
\hline Emamghorashi et al 35 & 2006 & Fars & 11 & 3011 & 9 & 3000 \\
\hline Esmaeili et al ${ }^{36}$ & 2007 & Bushehr & 72 & 19699 & 47 & 19627 \\
\hline Esmaieli et al ${ }^{37}$ & 2009 & Bushehr & 48 & 20342 & 42 & 20294 \\
\hline Etminan et $\mathrm{al}^{38}$ & 2019 & Kerman & 359 & 355507 & 139 & 355148 \\
\hline Farshadpour et al ${ }^{39}$ & 2016 & Khuzestan & 440 & 293894 & 295 & 293454 \\
\hline Ghafouri et $\mathrm{al}^{40}$ & 2011 & $\begin{array}{l}\text { South Kho- } \\
\text { rasan }\end{array}$ & 210 & 42862 & 13 & 42652 \\
\hline Ghavanini et $\mathrm{al}^{41}$ & 2000 & Fars & 85 & 7964 & 47 & 7879 \\
\hline Ghodsi Garamaleki et al ${ }^{42}$ & 2019 & $\begin{array}{c}\text { East Azarbaye- } \\
\text { jan }\end{array}$ & 279 & 216283 & & \\
\hline Habibzadeh et $\mathrm{al}^{43}$ & 2004 & Ardebil & 6 & 447 & 1 & 441 \\
\hline Hedayati-Moghaddam et al ${ }^{44}$ & 2019 & $\begin{array}{c}\text { Khorasan } \\
\text { Razavi }\end{array}$ & 227 & 58276 & 34 & 58049 \\
\hline Javadzadeh Shahshahani et al ${ }^{45}$ & 2013 & Yazd & 667 & 255427 & 239 & 254760 \\
\hline Karimi et al ${ }^{46}$ & 2008 & $\begin{array}{l}\text { Chaharmahal } \\
\text { \& Bakhtiari }\end{array}$ & 38 & 32162 & 74 & 32124 \\
\hline Kasraian et al ${ }^{47}$ & 2007 & Fars & 2499 & 510030 & 723 & 507531 \\
\hline Kasraian et al ${ }^{48}$ & 2008 & Fars & & & 203 & 93987 \\
\hline Kasraian et al ${ }^{49}$ & 2010 & Fars & 763 & 203761 & 391 & 202998 \\
\hline Kasraian et al ${ }^{50}$ & 2012 & Fars & 263 & 96909 & & \\
\hline Kazeminejad et al ${ }^{51}$ & 2005 & Golestan & 886 & 39806 & 74 & 38920 \\
\hline Khedmat et al ${ }^{52}$ & 2009 & Tehran & 5976 & 1010865 & 963 & 1004889 \\
\hline Maghsoodlu et al ${ }^{53}$ & 2018 & Kurdistan & 568 & 198136 & 103 & 197568 \\
\hline Mahdaviani et al ${ }^{54}$ & 2006 & Markazi & 80 & 11695 & 33 & 11615 \\
\hline Maleki et al ${ }^{55}$ & 2014 & Ilam & 29 & 4034 & 11 & 4005 \\
\hline Mansour Ghanaei et al ${ }^{56}$ & 2008 & Gilan & 997 & 222505 & 709 & 221508 \\
\hline
\end{tabular}




\begin{tabular}{|c|c|c|c|c|c|c|}
\hline \multirow{2}{*}{ Authors } & \multirow{2}{*}{ Year } & \multirow{2}{*}{ Province } & \multicolumn{2}{|c|}{ For HBV } & \multicolumn{2}{|c|}{ For HCV } \\
\hline & & & No. HBV & Total & No. HCV & Total \\
\hline Mardani et al ${ }^{57}$ & 2010 & Qom & 183 & 18032 & 47 & 17849 \\
\hline Masaeli et al ${ }^{58}$ & 2006 & Esfahan & 161 & 29619 & 72 & 29458 \\
\hline Mirrezai et al ${ }^{59}$ & 2014 & Tehran & 468 & 203099 & 66 & 202631 \\
\hline Mohammadali et al ${ }^{60}$ & 2014 & Tehran & 7869 & 2034497 & 2280 & 2026628 \\
\hline Mohammadi Tahroodi et al ${ }^{61}$ & 2018 & Ilam & 249 & 145522 & 66 & 145273 \\
\hline Mohsenizadeh et al ${ }^{62}$ & 2017 & Kerman & 524 & 99711 & 409 & 99187 \\
\hline Moniri et al ${ }^{63}$ & 2004 & Esfahan & 3 & 603 & 3 & 600 \\
\hline Nabavizadeh et al ${ }^{64}$ & 2000 & $\begin{array}{l}\text { Kohgiluyeh } \\
\text { and Boyer- } \\
\text { Ahmad }\end{array}$ & 48 & 5028 & 1 & 4980 \\
\hline Niazkar et al ${ }^{65}$ & 2020 & $\begin{array}{l}\text { Kohgiluyeh } \\
\text { and Boyer- } \\
\text { Ahmad }\end{array}$ & 247 & 198748 & 134 & 198501 \\
\hline Nour Kojory et al ${ }^{66}$ & 2007 & Mazandaran & 95 & 16789 & 15 & 16694 \\
\hline Omidkhoda et al ${ }^{67}$ & 2011 & Tehran & 54 & 11510 & 12 & 11456 \\
\hline Pourazar et al ${ }^{68}$ & 2006 & Esfahan & 346 & 51799 & 142 & 51453 \\
\hline Ranjbarian ${ }^{69}$ & 2007 & Hamedan & 46 & 8514 & & \\
\hline Rezaie et al ${ }^{70}$ & 2016 & Semnan & 100 & 42353 & 26 & 42253 \\
\hline Reza-Zadeh et al ${ }^{71}$ & 2006 & Hamedan & 141 & 18447 & 78 & 18306 \\
\hline Sajjadi et al ${ }^{72}$ & 2018 & $\begin{array}{l}\text { Kohgiluyeh } \\
\text { and Boyer- } \\
\text { Ahmad }\end{array}$ & 250 & 180554 & 115 & 180304 \\
\hline Sanei Moghaddam et al ${ }^{73}$ & 2004 & $\begin{array}{c}\text { Sistan and } \\
\text { Balouchestan }\end{array}$ & 168 & 7360 & 75 & 7192 \\
\hline Seyed Askari et al ${ }^{74}$ & 2015 & Kerman & 837 & 361559 & 292 & 360722 \\
\hline Sofian et al ${ }^{75}$ & 2010 & Markazi & 2 & 533 & 1 & 531 \\
\hline Sorouri Zanjani et al ${ }^{76}$ & 2013 & Zanjan & 104 & 29820 & 33 & 29716 \\
\hline Taheri Azbarmi et al ${ }^{77}$ & 2008 & Gilan & 130 & 49950 & 91 & 49820 \\
\hline Tajbakhsh et al ${ }^{78}$ & 2007 & $\begin{array}{l}\text { Chaharmahal } \\
\text { and Bakhtiari }\end{array}$ & & & 69 & 11472 \\
\hline Vahid et al ${ }^{79}$ & 2005 & Qazvin & 428 & 40026 & & \\
\hline Vossoughinia et al ${ }^{80}$ & 2010 & $\begin{array}{l}\text { Razavi Kho- } \\
\text { rasan }\end{array}$ & 3636 & 314154 & 311 & 310518 \\
\hline Yazhan et al ${ }^{81}$ & 2016 & $\begin{array}{l}\text { Razavi Kho- } \\
\text { rasan }\end{array}$ & 172 & 57507 & 13 & 57335 \\
\hline Zalei et $\mathrm{al}^{82}$ & 2017 & Kermanshah & & & 1 & 470 \\
\hline
\end{tabular}

470 to $1,137,582)$, lapsed $(1,640,069$ donors ranging from $3,463$ to 914,026$)$, and regular $(1,645,494$ donors ranged from 728 to $1,002,984$ ) blood donors, respectively.

13 studies $23,25-27,29,31,35,39,45,73,76,78,81$ had provided the number of $\mathrm{HCV}$ cases $(5,654 \mathrm{HCV}$ cases ranging from 6 to 3,071$)$ among male donors $(4,897,025$ male donors ranging from 2,787 to 2,240,419), and another 13 studies ${ }^{23,25-}$ $27,29,31,35,39,45,73,76,78,81$ had provided the number of HCV cases (172 HCV cases ranged from 0 to 75 ) among female donors
(327,142 female donors ranging from 213 to 172,856).

Using a random-effects model based on 58 studies, we found that the pooled prevalence of $\mathrm{HCV}$ was $0.22 \%(95 \%$ CI: $0.20-0.24$ ) in blood donors (figure $2 \mathrm{~B}$ ). There was significant inter-study heterogeneity across the included studies (I2: 98.64\%, $p<0.001)$. Meta-regression analysis results indicated that the date of publication $(\beta=-0.02, p<$ 0.001 ) was associated with HCV prevalence (figure $3 \mathrm{~B}$ ), but the total sample size did not show a significant impact 


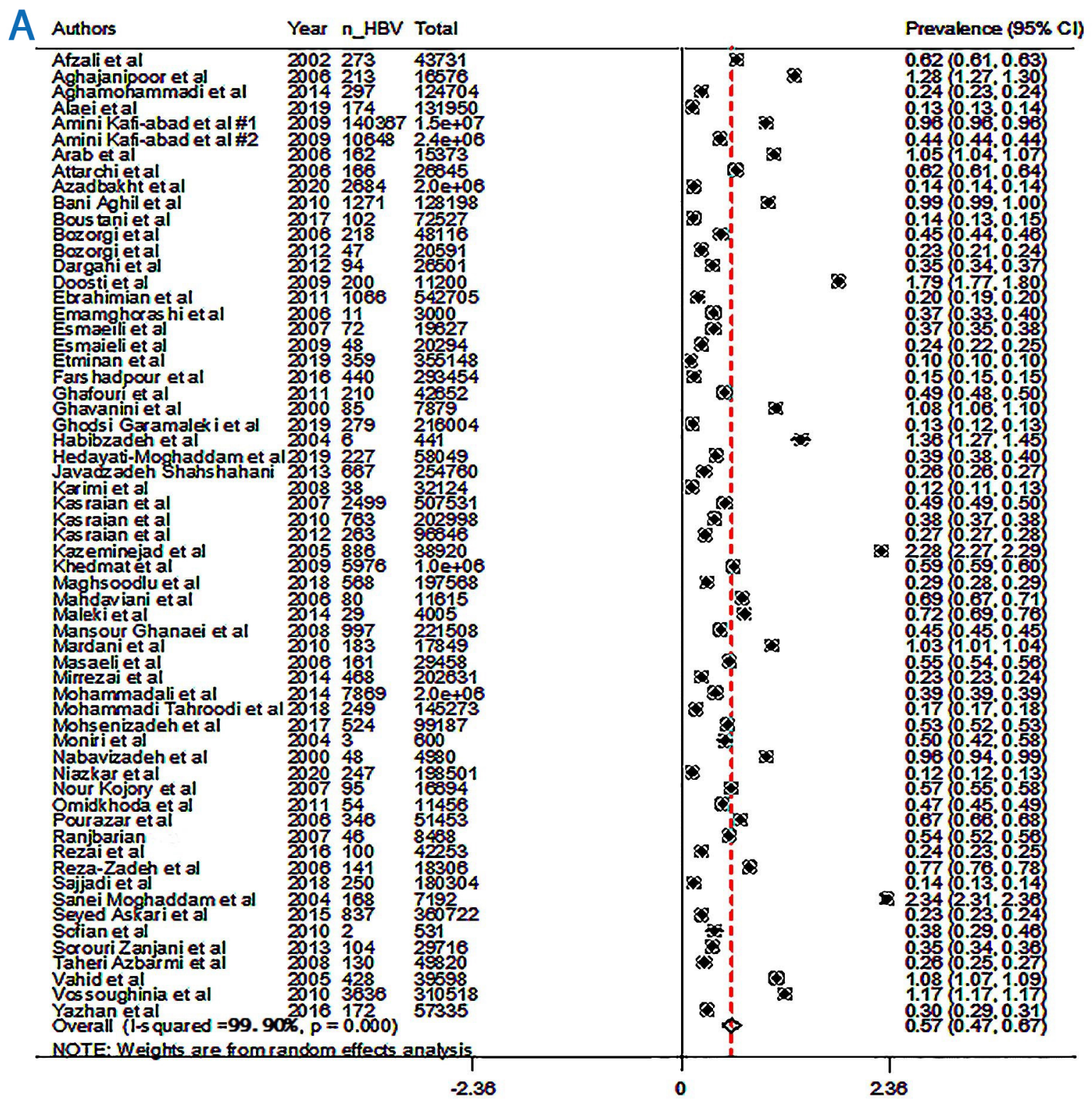

I^2 (variation in ES attributable to heterogeneity) $=$
Estimate of between-study variance Tau^2 =

99. $90 \%$

Test of $E S=0: z=$

$17.54 \mathrm{p}=$

0.00

. drop ES - _wT

Fig.2 A: The forest plot of pooled HBV

$(\beta=-0.00, p=0.115)$. The findings of sensitivity analysis for $\mathrm{HCV}$ showed that the highest pooled prevalence of HCV was $0.22 \%$ (95\% CI: $0.20-0.25$ ) after exclud- ing the study by Yazhan and colleagues. ${ }^{81}$ Also, the lowest pooled prevalence of HCV was $0.20 \%$ (95\% CI: $0.18-0.22)$ after excluding the study by Afzali and co-workers. ${ }^{19}$ The 


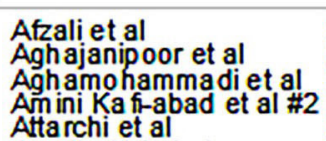

Attarchi et al

Azadbakht et al

2002477

43731
16576

201432 20093146 200642 2010161

Boustani et al

Bozorgi et al

Bozorgi et al

Delavan et al

Doosti et al

Ebrahimian et al

Emamghorashi et al

Esmaelli et al

Esmaieli et a

Etminan et al

Farshadpour et al

Ghafoun et al

Ghavanini et al

$\begin{array}{ll}1703 & 2.0 \mathrm{e}+06 \\ 161 & 128198\end{array}$

124704

$2.4 e+06$

201727

72527

201235

20126

200559

2011670

200747

200747

2019139

2016295

201113

200041

Hedayati-fl oghaddam

Javadzadeh Shahshaha

Karimi et al

Kasraian et al

Kasraian et a

Kasraian et al

Kazeminejad et al

Khedmat et a

if aghsoodlu et a

If ahdaviani et al

Iraleki et a

unsourGhanaei et al

Mardani et al

If asaeli et al

Mirrezai et al

Hohammadali et al

Hoh ammadi Tah roodi

Mohsenizadeh et al

Honiriet al

Nabavizadeh et al

Niazkar et al

Nour Kojory et al

Omidkhoda et al

Pourazaret al

Peurazar et

Rezai et al

Sajiadi et al

Saneill oghaddam et al

2013239

200874

2007723

2010391

200574

2009963

200633

201411

2008709

201047

200672

20142280

201866

2017409

20043

2000134

200715

2006142

201626

201626

2018115

Seyed Askari et al

Sofian et al

2015292

Sorouri Zanjani et al

Taheri Azbarmi et a

Tajbakhsh et a

Vossoughinia et al

Yazhan et al

48116

20591

15252

542705

3000

19627

20294

355148

42652

7879

441

58049

25476

32124

93987

902989

38920

$1.0 \mathrm{e}+06$
197568

11615

4005

221508

29458

29458

$2.0 \mathrm{e}+06$
145273

99187

600

4980

198501

1145

11456

42253

180304

7192

360722

(1)

201333

29716

2010311

201613

20171

11472
310518

57335

Overall (l-squared $=98.64 \%, p=0.000)$

NOTE Weights are fmm random effects analysis

4205.96 (d.f. $=57$ ) $p=$

$1.09(1.08,1.10)$
$0.48(0.46,0.49$

$0.03(0.02,0.03$

$0.160 .15,0.17$

$0.09\} 0.09,0.09$

$0.13\} 0.12,0.13$

$0.150 .14,0.16$

$0.170 .16,0.18$

$0.02(0.01,0.03$

$0.68(0.66,0.70$

$0.12(0.12,0.13\}$

$0.24\} 0.23,0.25$

$0.040 .04,0.04$

$0.10\} .10,0.10$

0.03 0.02, 0.04

$0.600 .57,0.62$

$0.06 \quad 0.05,0.07$

0.09 (09, 0.10

$0.2330 .22,0.24$

$0.20 .21,0.22$

0.19 0.19, 0.20

$0.19(0.18,0.20$

$0.100 .09,0.10\}$

$0.050 .05,0.06$

$0.270 .24,0.31$

$0.32,0.32,0.32\}$

$0.240 .23,0.26$

$0.03(0.03,0.04$

$0.110 .11,0.11$

$0.05\} 0.04,0.05$

$0.41\} 0.41,0.42$

0.02 (0.01. 0.05

$0.070 .06,0.07$

$0.0960 .07,0.11$

$0.103 .09,0.12$

$0.260 .27,0.28$

$0.430 .41,0.44$

$0.06<0.06,0.07$

- 1.04 1.02, 1.07

$0.0830 .08,0.08$

$011010,0.12$

$0.180 .17,0.19$

$0.180 .17,0.19$

$0.600 .58,0.62$

0.02 0.01, 0.03

$0.21(0.12,0.30\}$

Heterogeneity chi^2 =

ibutable to heterogeneity)

I^2 (variation in ES attributable to heter
Estimate of between-study variance Tau^2 $=$

0.00

98. 64\%

Test of $E S=0: z=$

$25.55 p=$

0. 00

Fig.2 B: The forest plot of pooled HCV prevalence 

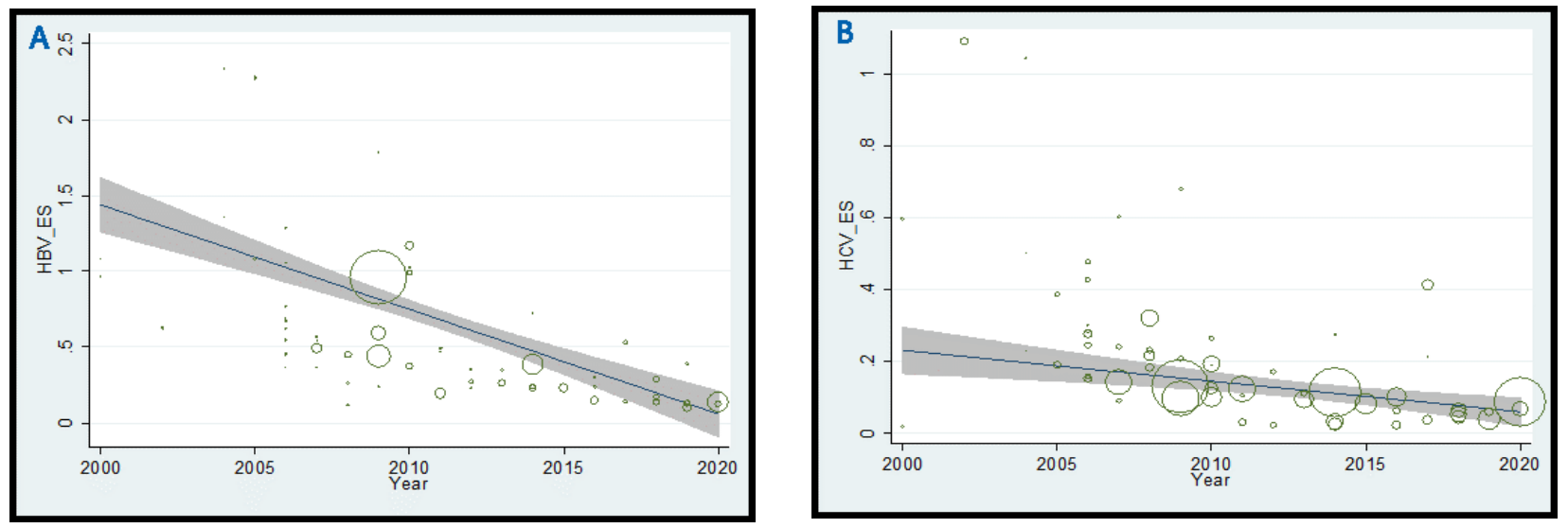

Fig. 3 A-B: Line graph of fitted values plotted using meta-regression against year for HBV (A) and HCV (B) prevalence

Table 2: The pooled prevalence of HBV and HCV based on blood donors' condition, sex, and quality of studies

\begin{tabular}{|c|c|c|c|c|c|}
\hline \multirow[b]{2}{*}{ Variables } & & \multicolumn{2}{|c|}{ HBV } & \multicolumn{2}{|c|}{$\mathrm{HCV}$} \\
\hline & & $\begin{array}{l}\text { No. of included } \\
\text { studies }\end{array}$ & $\begin{array}{l}\text { Pooled effect size } \\
(95 \% \text { CI })\end{array}$ & $\begin{array}{l}\text { No. of included } \\
\text { studies }\end{array}$ & $\begin{array}{l}\text { Pooled effect size } \\
(95 \% \text { CI })\end{array}$ \\
\hline \multirow{3}{*}{$\begin{array}{l}\text { Donors' } \\
\text { condition }\end{array}$} & First time blood donors & 15 & $1.06 \%(0.90-1.22)$ & 14 & $0.43 \%(0.37-0.48)$ \\
\hline & Lapsed blood donors & 14 & $0.19 \%(0.14-0.24)$ & 12 & $0.13 \%(0.10-0.17)$ \\
\hline & Regular blood donors & 10 & $0.33 \%(0.27-0.39)$ & 9 & $0.28 \%(0.24-0.33)$ \\
\hline \multirow{2}{*}{ Sex } & Male & 15 & $0.57 \%(0.44-0.70)$ & 13 & $0.22 \%(0.19-0.25)$ \\
\hline & Female & 15 & $0.44 \%(0.45-0.53)$ & 13 & $0.23 \%(0.16-0.30)$ \\
\hline \multirow{2}{*}{ Quality status } & Low quality & 23 & $0.75 \%(0.51-0.99)$ & 23 & $0.33 \%(0.20-0.46)$ \\
\hline & High quality & 38 & $0.46 \%(0.34-0.59)$ & 35 & $0.15 \%(0.13-0.17)$ \\
\hline
\end{tabular}

results of the subgroup analyses are presented in table 2.

\section{Quality assessment}

High-quality studies related to the prevalence of $\mathrm{HBV}$ and HCV among blood donors were 38 and 35, respectively. The results of subgroup analyses according to the quality of studies are summarized in table 2. Quality indicators of different studies and details of the scoring method are also presented in table S1.

\section{DISCUSSION}

The first global health sector strategy (GHSS) on viral hepatitis was prepared in May 2016 by the World Health Assembly, the decision-making body of WHO. The program requires the members to eliminate viral hepatitis as a public health threat by 2030 aiming to reduce new infections by $90 \%$ and mortality by $65 \%$. Blood and injection safety is the core of the intervention of the elimination program. $^{2}$

$\mathrm{HBV}$ and $\mathrm{HCV}$ were responsible for $96 \%$ of all mortality causes related to hepatitis. In addition, most hepatitis deaths in 2015 were commonly attributed to cirrhosis (720,000 deaths) and HCC (470,000 deaths). The global prevalence of $\mathrm{HBV}$ infection was $3.5 \%$ in the general population, and about 257 million persons have chronic infection with HBV. WHO African region and the Western Pacific region have the highest reported prevalence of $\mathrm{HBV}^{2}{ }^{2}$ Blood donors are among the high-risk populations with an increased incidence of HBV infection. WHO recommends screening of all blood donations for evidence of infections such as HBV. ${ }^{3}$ Blood transfusion is an uncommon event in a person's life; hence, contaminated transfusion is not a major source of viral hepatitis transmission compared with other risk factors such as unsafe injections. ${ }^{83,84}$ However, WHO actively recruits VNRDs for the promotion of blood safety because paid donors had a higher prevalence of blood-borne pathogens. ${ }^{5,85}$

Regarding the result of the present investigation, the pooled prevalence of $\mathrm{HBV}$ was $0.57 \%$ among blood donors, which was in the range of the previous report. Babanejad 
Table S1: Quality Indicators from Newcastle-Ottawa Scale

\begin{tabular}{|c|c|c|c|c|c|c|c|c|c|c|c|c|c|}
\hline & Authors & Year & 1 & 2 & 3 & $4 \mathrm{~A}$ & $4 B$ & $\mathbf{5 A}$ & 5B & 6 & 7 & Stars & Quality \\
\hline 1 & Afzali et al (1) & 2002 & $*$ & $*$ & $*$ & & & $*$ & $*$ & & & 5 & Low \\
\hline 2 & Aghajanipoor et al (2) & 2006 & * & * & $*$ & & & $*$ & $*$ & $*$ & & 6 & Low \\
\hline 3 & Aghamohammadi et al (3) & 2014 & $*$ & * & $*$ & & & $*$ & $*$ & $*$ & * & 7 & High \\
\hline 4 & Alaei et al (4) & 2019 & $*$ & $*$ & $*$ & & & $*$ & $*$ & $*$ & $*$ & 7 & High \\
\hline 5 & Amini Kafi-abad et al \#1 (5) & $2009 a$ & $*$ & $*$ & $*$ & & & $*$ & $*$ & $*$ & $*$ & 7 & High \\
\hline 6 & Amini Kafi-abad et al \#2 (6) & $2009 b$ & $*$ & $*$ & $*$ & & & $*$ & $*$ & $*$ & $*$ & 7 & High \\
\hline 7 & Arab et al (7) & 2006 & $*$ & $*$ & $*$ & & & $*$ & $*$ & $*$ & & 6 & Low \\
\hline 8 & Attarchi et al (8) & 2006 & $*$ & $*$ & $*$ & & & $*$ & $*$ & $*$ & & 6 & Low \\
\hline 9 & Azadbakht et al (9) & 2020 & $*$ & * & $*$ & & & $*$ & $*$ & $*$ & $*$ & 7 & High \\
\hline 10 & Bani Aghil et al (10) & 2010 & $*$ & $*$ & $*$ & & & $*$ & $*$ & $*$ & $*$ & 7 & High \\
\hline 11 & Boustani et al (11) & 2017 & $*$ & * & $*$ & & & $*$ & $*$ & $*$ & $*$ & 7 & High \\
\hline 12 & Bozorgi et al (12) & 2006 & $*$ & * & $*$ & & & $*$ & $*$ & $*$ & $*$ & 7 & High \\
\hline 13 & Bozorgi et al (13) & 2012 & $*$ & $*$ & $*$ & & & $*$ & $*$ & $*$ & $*$ & 7 & High \\
\hline 14 & Dargahi et al (14) & 2012 & $*$ & $*$ & $*$ & & & $*$ & $*$ & & & 5 & Low \\
\hline 15 & Delavari et al (15) & 2005 & $*$ & $*$ & $*$ & & & * & $*$ & & $*$ & 6 & Low \\
\hline 16 & Doosti et al (16) & 2009 & $*$ & $*$ & * & & & $*$ & $*$ & & $*$ & 6 & Low \\
\hline 17 & Ebrahimian et al (17) & 2011 & $*$ & * & $*$ & & & $*$ & $*$ & $*$ & $*$ & 7 & High \\
\hline 18 & Emamghorashi et al (18) & 2006 & $*$ & * & $*$ & & & $*$ & $*$ & $*$ & & 6 & Low \\
\hline 19 & Esmaeili et al (19) & 2007 & $*$ & * & $*$ & & & $*$ & $*$ & $*$ & & 6 & Low \\
\hline 20 & Esmaieli et al (20) & 2009 & $*$ & $*$ & $*$ & & & $*$ & $*$ & $*$ & & 6 & Low \\
\hline 21 & Etminan et al (21) & 2019 & $*$ & $*$ & $*$ & & & $*$ & * & $*$ & $*$ & 7 & High \\
\hline 22 & Farshadpour et al (22) & 2016 & $*$ & $*$ & $*$ & & & * & $*$ & $*$ & $*$ & 7 & High \\
\hline 23 & Ghafouri et al (23) & 2011 & $*$ & $*$ & $*$ & & & $*$ & $*$ & $*$ & $*$ & 7 & High \\
\hline 24 & Ghavanini et al (24) & 2000 & $*$ & $*$ & $*$ & & & * & $*$ & $*$ & $*$ & 7 & High \\
\hline 25 & Ghodsi Garamaleki et al (25) & 2019 & $*$ & * & $*$ & & & $*$ & $*$ & & & 5 & Low \\
\hline 26 & Habibzadeh et al (26) & 2004 & $*$ & & $*$ & & & $*$ & $*$ & & & 4 & Low \\
\hline 27 & Hedayati-Moghaddam et al (27) & 2019 & $*$ & $*$ & $*$ & & & $*$ & * & $*$ & & 6 & Low \\
\hline 28 & Javadzadeh Shahshahani et al (28) & 2013 & $*$ & $*$ & * & & & $*$ & $*$ & $*$ & $*$ & 7 & High \\
\hline 29 & Karimi et al (29) & 2008 & $*$ & $*$ & $*$ & & & * & $*$ & & $*$ & 6 & Low \\
\hline 30 & Kasraian et al (30) & 2007 & * & $*$ & * & & & $*$ & $*$ & * & $*$ & 7 & High \\
\hline 31 & Kasraian et al (31) & 2008 & $*$ & * & $*$ & $*$ & * & $*$ & $*$ & $*$ & $*$ & 9 & High \\
\hline 32 & Kasraian et al (32) & 2010 & $*$ & $*$ & $*$ & & & $*$ & $*$ & $*$ & * & 7 & High \\
\hline 33 & Kasraian et al (33) & 2012 & $*$ & $*$ & $*$ & & & $*$ & $*$ & $*$ & $*$ & 7 & High \\
\hline 34 & Kazeminejad et al (34) & 2005 & $*$ & * & $*$ & & & $*$ & $*$ & & $*$ & 6 & Low \\
\hline 35 & Khedmat et al (35) & 2009 & $*$ & * & $*$ & & & $*$ & $*$ & $*$ & * & 7 & High \\
\hline 36 & Maghsoodlu et al (36) & 2018 & $*$ & $*$ & $*$ & & & $*$ & $*$ & $*$ & $*$ & 7 & High \\
\hline 37 & Mahdaviani et al (37) & 2006 & $*$ & $*$ & $*$ & & & $*$ & $*$ & $*$ & $*$ & 7 & High \\
\hline 38 & Maleki et al (38) & 2014 & $*$ & $*$ & $*$ & & & $*$ & $*$ & $*$ & $*$ & 7 & High \\
\hline 39 & Mansour Ghanaei et al (39) & 2008 & $*$ & $*$ & $*$ & & & $*$ & $*$ & $*$ & $*$ & 7 & High \\
\hline 40 & Mardani et al (40) & 2010 & $*$ & $*$ & $*$ & & & $*$ & $*$ & $*$ & * & 7 & High \\
\hline 41 & Masaeli et al (41) & 2006 & $*$ & $*$ & $*$ & & & * & $*$ & $*$ & $*$ & 7 & High \\
\hline 42 & Mirrezai et al (42) & 2014 & $*$ & $*$ & $*$ & $*$ & $*$ & $*$ & $*$ & $*$ & $*$ & 9 & High \\
\hline 43 & Mohammadali et al (43) & 2014 & * & * & * & & & * & $*$ & $*$ & * & 7 & High \\
\hline
\end{tabular}




\begin{tabular}{|c|c|c|c|c|c|c|c|c|c|c|c|c|c|}
\hline & Authors & Year & 1 & 2 & 3 & $4 \mathrm{~A}$ & 4B & $\mathbf{5 A}$ & 5B & 6 & 7 & Stars & Quality \\
\hline 44 & Mohammadi Tahroodi et al (44) & 2018 & * & * & $*$ & & & * & $*$ & & $*$ & 6 & Low \\
\hline 45 & Mohsenizadeh et al (45) & 2017 & $*$ & $*$ & $*$ & & & $*$ & $*$ & $*$ & $*$ & 7 & High \\
\hline 46 & Moniri et al (46) & 2004 & $*$ & & $*$ & & & $*$ & $*$ & $*$ & & 5 & Low \\
\hline 47 & Nabavizadeh et al (47) & 2000 & $*$ & $*$ & $*$ & & & $*$ & $*$ & & & 5 & Low \\
\hline 48 & Niazkar et al (48) & 2020 & $*$ & $*$ & * & & & $*$ & $*$ & $*$ & $*$ & 7 & High \\
\hline 49 & Nour Kojory et al (49) & 2007 & $*$ & $*$ & $*$ & & & $*$ & $*$ & $*$ & $*$ & 7 & High \\
\hline 50 & Omidkhoda et al (50) & 2011 & $*$ & $*$ & $*$ & $*$ & * & $*$ & $*$ & $*$ & $*$ & 9 & High \\
\hline 51 & Pourazar et al (51) & 2006 & $*$ & $*$ & $*$ & & & * & $*$ & $*$ & * & 7 & High \\
\hline 52 & Ranjbarian (52) & 2007 & $*$ & * & $*$ & & & $*$ & $*$ & $*$ & & 6 & Low \\
\hline 53 & Rezaie et al (53) & 2016 & $*$ & $*$ & $*$ & & & $*$ & $*$ & $*$ & $*$ & 7 & High \\
\hline 54 & Reza-Zadeh et al (54) & 2006 & $*$ & $*$ & $*$ & & & * & $*$ & & & 5 & Low \\
\hline 55 & Sajjadi et al (55) & 2018 & $*$ & $*$ & * & & & $*$ & $*$ & $*$ & $*$ & 7 & High \\
\hline 56 & Sanei Moghaddam et al (56) & 2004 & $*$ & * & * & & & * & $*$ & $*$ & & 6 & Low \\
\hline 57 & Seyed Askari et al (57) & 2015 & $*$ & $*$ & $*$ & & & $*$ & $*$ & $*$ & $*$ & 7 & High \\
\hline 58 & Sofian et al (58) & 2010 & $*$ & & $*$ & & & $*$ & $*$ & $*$ & $*$ & 6 & Low \\
\hline 59 & Sorouri Zanjani et al (59) & 2013 & $*$ & $*$ & $*$ & & & * & $*$ & & $*$ & 6 & Low \\
\hline 60 & Taheri Azbarmi et al (60) & 2008 & $*$ & $*$ & $*$ & & & $*$ & $*$ & & $*$ & 6 & Low \\
\hline 61 & Tajbakhsh et al (61) & 2007 & $*$ & $*$ & $*$ & & & $*$ & $*$ & $*$ & & 6 & Low \\
\hline 62 & Vahid et al (62) & 2005 & $*$ & $*$ & $*$ & $*$ & * & * & $*$ & $*$ & $*$ & 9 & High \\
\hline 63 & Vossoughinia et al (63) & 2010 & $*$ & $*$ & $*$ & $*$ & & * & $*$ & $*$ & & 7 & High \\
\hline 64 & Yazhan et al (64) & 2016 & $*$ & * & $*$ & & & $*$ & $*$ & $*$ & $*$ & 7 & High \\
\hline 65 & Zalei et al (65) & 2017 & * & & * & & & * & $*$ & $*$ & * & 6 & Low \\
\hline
\end{tabular}

and colleagues reported that the pooled prevalence of HBsAg in blood donors in the WHO Eastern Mediterranean Region (EMRO) and exclusively in Iran was 1.99\% and $0.58 \%$, respectively. ${ }^{3}$ Throughout the last two decades, HBV prevalence was declined in Iran as a result of designing and running a national vaccination program in infants, mandatory screening of pregnant women, proper treatment of newborns delivered by infected mothers, increasing the knowledge of people toward the routes of HBV transmission and its risk factors, and finally vaccination of high-risk groups especially health care workers. ${ }^{8,86-88}$ Previous reports reveal that Iran is classified as one of the countries with a low-intermediate prevalence of HBV (2-4.9\%). ${ }^{89}$ Since many positive cases with viral hepatitis are excluded from donating blood, the prevalence of HBsAg in blood donors is not an indicator of the prevalence among the whole population. This means that the donor population is representative of low-risk individuals prone to viral hepatitis infection via specific means, including contamination of the equipment or other means that are usually underestimated. , $^{3,13,86}$
It is suggested that 1.75 million new $\mathrm{HCV}$ infections occurred worldwide in 2015, and the global prevalence of this infection is about $1 \%$, according to the literature . The incidence and prevalence rates of $\mathrm{HCV}$ infection were reported to be 62.5 per 100000 (total number: 409000) and 2.3\% (total number: 15 million) in EMRO, respectively. Although the prevalence of $\mathrm{HCV}$ is lower than HBV, the infection is distributed more heterogeneously in Iran than in the world. ${ }^{2}$ The prevalence of $\mathrm{HCV}$ in Iran has been reported to be $0.6 \%$ in the general population. ${ }^{14}$ Therefore, Iran could be categorized as a low-prevalent country for $\mathrm{HCV}$ infection..$^{90}$ Moreover, Iran has the lowest rate of $\mathrm{HCV}$ infection compared with most of the other countries in the Middle-East region. ${ }^{13,15}$ However, a recent increase was seen in the patients affected by HCV infection, and probably this type of hepatitis will replace $\mathrm{HBV}$ in the near future as the most common cause of the chronic liver disease. ${ }^{91,92}$ We conclude that the pooled prevalence of $\mathrm{HCV}$ is $0.22 \%$ among blood donors in Iran, which is much lower than previous studies in which $0.5 \%$ of blood donors were infected with $\mathrm{HCV}{ }^{15}$ 
The present study shows that the prevalence of HBV in Iran is non-uniformly distributed. This was ranged between $0.1 \%$ in Kerman to $2.34 \%$ in Sistan and Baluchestan provinces, both located in the southeastern part of the country. ${ }^{38,73}$ Another result of the current analysis is the relatively heterogeneous dispersion of $\mathrm{HCV}$ prevalence from $0.02 \%$ in Khorasan Razavi and Kohgiluyeh and BoyerAhmad provinces, located in central-western and northeastern parts of Iran to $1.09 \%$ in Esfahan, in the center of Iran .19,64,81 This might be due to significant differences in the quality of public health, habits, risk factors, and lifestyles in various geographical regions.$^{15}$ The year of publication of data and the geographical location were also probable sources of heterogeneity among high-risk groups infected with both $\mathrm{HBV}$ and $\mathrm{HCV} .{ }^{93,94}$

In the end, some limitations of this study should be mentioned. First, over one-third of the studies included in this review had low quality. Second, the reported age of the individuals was diverse, so we were not able to report the findings by age classification. It is suggested that future studies focus on standardized formulae for sample size calculation and report of definite age groups for better estimation of the prevalence of viral hepatitis in blood donors to inform policymakers and public health providers.

\section{CONCLUSION}

The results of the present study indicate that the prevalence of $\mathrm{HBV}$ and $\mathrm{HCV}$ decreased steadily among blood donors in Iran during the past two decades. The policies toward controlling the prevalence of viral hepatitis seem to be relatively efficient, although lowering the rate is still an important concern.

\section{ACKNOWLEDGMENTS}

The authors want to thank Dr. Soheil Ashkani Esfahani, Medipress $^{\mathrm{TM}}$, and SIMR Co. for providing the required data and assisting in preparing the draft of the present paper and scientific editing of the paper. The authors would also like to thank the Center for Development of Clinical Research of Nemazee Hospital and Dr. Nasrin Shokrpour for editorial assistance. The authors also thank Dr. Mohammad Salehi Marzijarani for his cooperation in analyzing the data.

\section{Statement of Ethics}

The protocol of this study was approved by the Ethics Committee, managed by Prof. S.Z. Tabei, at Shiraz University of Medical Sciences, Shiraz, Iran.

\section{Disclosure Statement}

The authors declare that they have no competing interests. All the expenditure was provided by the authors, and nothing was received from any other source or organization.

\section{Authors' Contribution}

LK and SH gathered the data and extracted the data out of the papers, and classified them for analysis. RT performed the data analysis and prepared the results of the paper out of raw data. SH, RSR, AE, and MHI prepared the draft, edited it, and finalized the paper. All authors reviewed the final draft of the paper.

\section{ETHICAL APPROVAL}

There is nothing to be declared.

\section{CONFLICT OF INTEREST}

The authors declare no conflict of interest related to this work.

\section{REFERENCES}

1. Stasi C, Silvestri C, Voller F, Cipriani F. The epidemiological changes of HCV and HBV infections in the era of new antiviral therapies and the anti-HBV vaccine. $J$ Infect Public Health 2016;9:389-95. doi: 10.1016/j. jiph.2015.05.004.

2. WHO. Global Hepatitis Report 2017. Geneva: World Health Organization; 2017. Licence: CC BY-NC-SA. 2017;3.

3. Babanejad M, Izadi N, Najafi F, Alavian SM. The HBsAg Prevalence among blood donors from Eastern Mediterranean and Middle Eastern countries: a systematic review and meta-Analysis. Hepat Mon 2016;16:e35664. doi: 10.5812/ hepatmon.35664

4. WHO-Expert-Group. Expert Consensus Statement on achieving self-sufficiency in safe blood and blood products, based on voluntary non-remunerated blood donation (VNRBD). Vox Sang 2012;103:337-42. doi: 10.1111/j.14230410.2012.01630.x

5. Asenso-Mensah K, Achina G, Appiah R, Owusu-Ofori S, Allain JP. Can family or replacement blood donors become regular volunteer donors? Transfusion 2014;54:797-804. doi: $10.1111 / \operatorname{trf} .12216$ 
6. Amini Kafi-Abad S, Rezvan H, Abolghasemi H. Trends in prevalence of hepatitis B virus infection among Iranian blood donors, 1998-2007. Transfus Med 2009;19:189-94. doi: $10.1111 /$ j.1365-3148.2009.00935.x.

7. Alavian SM, Fallahian F, Lankarani KB. The changing epidemiology of viral hepatitis B in Iran. $J$ Gastrointestin Liver Dis 2007;16:403-6.

8. Salehi-Vaziri M, Sadeghi F, Hashiani AA, Fesharaki MG, Alavian SM. Hepatitis B virus infection in the general population of Iran: an updated systematic review and meta-analysis. Hepat Mon 2016;16:e35577. doi: 10.5812/hepatmon.35577.

9. Cheraghali A. Overview of blood transfusion system of iran: 2002-2011. Iran J Public Health 2012;41:89-93.

10. European-Association-for-Study-of-Liver. EASL Clinical Practice Guidelines: management of hepatitis $\mathrm{C}$ virus infection. J Hepatol 2014;60:392-420. doi: 10.1016/j. jhep.2013.11.003.

11. Lorenzo CD, Angus AG, Patel AH. Hepatitis C virus evasion mechanisms from neutralizing antibodies. Viruses 2011;3:2280-300. doi: 10.3390/v3112280.

12. Amiri FB, Mostafavi E, Mirzazadeh A. HIV, HBV and $\mathrm{HCV}$ coinfection prevalence in Iran-a systematic review and meta-analysis. PloS One 2016;11:e0151946. doi: 10.1371/journal.pone.0151946. eCollection 2016.

13. Taherkhani R, Farshadpour F. Epidemiology of hepatitis $C$ virus in Iran. World J Gastroenterol 2015;21:10790-810. doi: 10.3748/wjg.v21.i38.10790.

14. Mirminachi B, Mohammadi Z, Merat S, Neishabouri A, Sharifi AH, Alavian SH, et al. Update on the prevalence of hepatitis $\mathrm{C}$ virus infection among Iranian general population: a systematic review and meta-analysis. Hepat Mon 2017;17:e42291. doi: 10.5812/hepatmon.42291.

15. Khodabandehloo M, Roshani D, Sayehmiri K. Prevalence and trend of hepatitis $\mathrm{C}$ virus infection among blood donors in Iran: A systematic review and meta-analysis. J Res Med Sci 2013;18:674-82.

16. Moher D, Liberati A, Tetzlaff J, Altman DG. Preferred reporting items for systematic reviews and meta-analyses: the PRISMA statement. PLoS Med 2009;6:e1000097. doi: 10.1371/journal.pmed.1000097.

17. Bigna JJ, Amougou MA, Asangbeh SL, Kenne AM, Nansseu JR. Seroprevalence of hepatitis C virus infection in Cameroon: a systematic review and meta-analysis. BMJ Open 2017;7:e015748. doi: 10.1136/bmjopen-2016-015748.

18. Nyaga VN, Arbyn M, Aerts M. Metaprop: a Stata command to perform meta-analysis of binomial data. Arch Public Health 2014;72:39. doi: 10.1186/2049-3258-72-39. eCollection 2014

19. Afzali H, Taghavi-Ardakani A, Vali G. Sero-epidemiology of hepatitis B and C in blood donors in Kashan 1375-1380 (1996-2001) (in Persian). Feyz 2002;6:43-50.

20. Aghajanipoor K, Zandieh T. Seroepidemiological investigation of hepatitis B,C and HIV virus in safe blood donors of Babol Blood Transfusion Center. Sci J Iran Blood Transfus Organ 2006;2:339-41.
21. Aghamohamadi A, Montazeri M, Akbari M. Prevalence of hepatitis $\mathrm{B}$ and hepatitis $\mathrm{C}$ in blood donors at Semnan province from 2008 to 2011. Faslnamahi Kumish 2014;15:162-7.

22. Alaei M, Zadsar M, Pouriani E. The prevalence and probable risk factors of HbsAg positive blood donors in Mazandaran province in 2013. Sci J Iran Blood Transfus Organ 2019;16:241-50

23. Amini Kafi-abad S, Rezvan H, Abolghasemi H, Talebian A. Prevalence and trends of human immunodeficiency virus, hepatitis $\mathrm{B}$ virus, and hepatitis $\mathrm{C}$ virus among blood donors in Iran, 2004 through 2007. Transfusion 2009;49:2214-20. doi: $10.1111 /$ j.1537-2995.2009.02245.x.

24. Arab M, Abas-zadeh A, Pourabuli B, Soleimanizadeh L, Shahsavari M, Javadi M. Prevalence of HBsAg positivity in blood donors in Bam, 1999-2002. Sci J Iran Blood Transfus Organ 2006;3:277-80.

25. Attarchi Z, Ghafouri M, Hajibaygi B, Assari S, Alavian SM. Donor deferral and blood-borne infections in blood donors of Tehran. Sci J Iran Blood Trans Organ 2006;2:353-64.

26. Azadbakht M, Ardakani MT, Delirakbariazar M, Kasraian L, Khaledi A, Foruozandeh H, et al. Seroprevalence and Trend of HBV, HCV, and HIV Infections among Blood Donors of Fars Province, Iran (2006-2018). Ethiop $J$ Health Sci 2020;30:397-407. doi: 10.4314/ejhs.v30i3.11.

27. Bani Aghil SS, Abbasi S, Arab M, Seyedein MS. The Prevalence of HCV, HBV, HIV in Blood Donors of Golestan Province, (2006-2008). Med Lab J 2009;3:1-5.

28. Boustani H, Anvari E, Saiadi Sartang S, Omidi M, Rostami E, Mohamadi Z. Prevalence of HIV, hepatitis B and $\mathrm{C}$ infections among volunteer blood donors at the blood transfusion center of Ilam city, Iran. J Basic Res Med Sci 2017;4:4-8. doi: 10.18869/acadpub.jbrms.4.2.4

29. Bozorgi SH, Ramezani H, Nooranipour M, Ahmadi M, Baghernejad A, Mostajeri A, et al. Risk factors of viral hepatitis: yet to explore. Transfus Apher Sci 2012;47:1459. doi: 10.1016/j.transci.2012.06.023.

30. Bozorgi S, AhmadzadAsl M, Ramezani H, Kargarfard H, Alavian S. Study of Viral Infections Prevalence in Blood Donors of Qazvin Province in Different Time Intervals and During Bam Earthquake. Govaresh 2006;11:242-8.

31. Dargahi F, Amani F, Habibzadeh S, Ezzati M, Sadri Z. The epidemiology of HCV, HBV, HIV, in blood donors in Ardabil province in 1389 (2010) (In Persian). Caduceus 2012;2:31-8

32. Delavari M, Tabatabaie S, Sheikh Bardsiri H, Maarefdust $\mathrm{Z}$, Zandieh T. The prevalence of Hepatitis $\mathrm{C}$ and its related factors among blood donors of Kerman Blood Center. Sci J Iran Blood Transfus Organ 2006;2:269-71.

33. Doosti A, Amini-Bavil-Olyaee S, Tajbakhsh E, Adeli A, Mahboudi F. Prevalence of viral hepatitis and molecular analysis of HBV among voluntary blood donors in west Iran. New Microbiol 2009;32:193-8.

34. Ebrahimian Z, Fazilati M, Akbari N, Hariri MM, Fatehifar MR. Correlation of deferral rate with the frequency rate of viral markers of HBV, HCV and HIV in blood supplies 
during 2004 to 2009. Sci J Iran Blood Transfus Organ 2011;8:130-6.

35. Emamghoreishi F, Fathi GH, Mohtashami A. Evaluation of demographic characteristics and hepatitis B,C and HIV prevalence among blood donors in Jahrom. Sci J Iran Blood Transfus Organ 2006;2:373-8.

36. Esmaieli H, Hajiani G, Esmailie M, Monkhian A, Azizzadeh M, Hamidia Z. Evaluation of infection with hepatitis B, C, $\mathrm{HIV}$ and syphilis among blood donors in Bushehr province in 2005. Iran J Infect Dis Trop Med 2007;12:85-6.

37. Esmaieli H, Hajiani G, Mankhian A, Pourmehdi Broujeni M. Seroepidemiological survey of hepatitis B, C, HIV and syphilis among blood donors in Bushehr-Iran (in Persian). Iran South Med J 2009;11:183-90.

38. Etminan A, Naghibzadeh-Tahami A, Askari S. The association between the prevalence of transfusion transmitted infections and characteristics of infected blood donors in kerman, Iran. J Kerman Uni Med Sci 2019;26:377-83.

39. Farshadpour F, Taherkhani R, Tajbakhsh S, Gholizadeh Tangestani M, Hajiani G, Sharifi N, et al. Prevalence and Trends of Transfusion-Transmissible Viral Infections among Blood Donors in South of Iran: An Eleven-Year Retrospective Study. PloS One 2016;11:e0157615. doi: 10.1371/journal.pone.0157615. eCollection 2016.

40. Ghafouri M, Ameli M. Comparing prevalence of transfusion transmitted viral infections in various population groups of South Khorasan. Sci J Iran Blood Transfus Organ 2011;7:242-8.

41. Ghavanini AA, Sabri MR. Hepatitis B surface antigen and anti-hepatitis $\mathrm{C}$ antibodies among blood donors in the Islamic Republic of Iran. East Mediterr Health $J$ 2000;6:1114-6.

42. Ghodsi Garamaleki M, Ahmadizadeh C. Prevalence of hepatitis B among blood donors referring to blood transfusion organization: brief report. Tehran Uni Med J 2019;76:699-702.

43. Habibzadeh S, Davarnia B, Bazazataei A, Bagherzadeh S, Hamid Kholgh GR. Epidemiological evaluation of transfusion transmitted diseases in Ardabil in Tasoua and Ashoura 2003. Sci J Iran Blood Transfu Organ 2005;1:55-60.

44. Hedayati-Moghaddam MR, Foomani FM, Gowhari Shabgah A. Frequency of Viral Transfusion-Transmitted Infections (TTIs) Among Resident and Pilgrim Blood Donors in Mashhad, 2011. Int J Infect 2019;6:e87350. doi: 10.5812/ iji.87350.

45. Javadzadeh Shahshahani H, Vaziri M, Mansouri F. Seven years trends in prevalence of transfusion-transmissible viral infections in Yazd blood transfusion organization. Iran $J$ Ped Hematol Oncol 2013;3:119-24.

46. Karimi A, Hoseini SM. Seroprevalence of hepatitis B and C virus and HIV markers among blood donors from ShahreKord, Iran (2004-2006). Kuwait Med J 2008;40:285-7.

47. Kasraian L, Jahromi T, Ardeshir S. Prevalence of major transfusion transmitted viral infections (HCV, HBV, HIV) in Shiraz blood donors from 2000 to 2005. Sci J Iran Blood Transfus Organ 2007;3:373-8.
48. Kasraian L, Tavassoli AR. Prevalence of hepatitis C and its risk factors in blood donors at Shiraz transfusion center. Faslnamahi Kumish 2008;10:7-12.

49. Kasraian L, Tavasoli A. Positivity of HIV, hepatitis B and hepatitis $\mathrm{C}$ in patients enrolled in a confidential self-exclusion system of blood donation: a cross-sectional analytical study. Sao Paulo Med J 2010;128:320-3. doi: 10.1590/s151631802010000600002 .

50. Kasraian L, Tavassoli A, Shayegan M, Alavian SM. The prevalence and risk factor of hepatitis B and D in Shiraz blood donors. Afr J Microbiol Res 2012;6:3976-9. doi: 10.5897/AJMR11.822.

51. Kazeminejad V, Azarhoosh R, Moulana AA, Dehbashi GR. Frequency of hepatitis $\mathrm{B}, \mathrm{C}$ and $\mathrm{HIV}$ in blood donors and patients referring to Gorgan Blood Transfusion Organization (2003). J Gorgan Univ Med Sci 2005;7:84-6.

52. Khedmat H, Alavian SM, Miri SM, Amini M, Aboighasemi H, Hajibeigi B, et al. Trends in Seroprevalence of Hepatitis B, Hepatitis C, HIV, and Syphilis Infections in Iranian Blood Donors from 2003 to 2005. Hepat Mon 2009;9:24-8.

53. Maghsoodlu M, Salehifar P, Rahimzadeh P, Babahajian W, Mohammadi S, Babahajian S, et al. Prevalence and Trends of Transfusion-Transmissible Infections and Study of Confidential Unit Exclusion among Blood Donors in Kurdistan Province of Iran. Int J Med Lab 2018;5:58-65.

54. Mahdaviani F, Maghsoudlu M, Pourfathollah AA. Prevalence of blood transmitted viral infections in regular and nonregular donors of Arak Blood Center. Sci J Iran Blood Trans Organ 2006;2:343-51.

55. Maleki F, Hemati S, Mahdavi Z, Azizian M, Amraei M. Prevalence of viral infection of blood donors in Ilam blood transfusion center. J Basic Res Med Sci 2014;1:51-5.

56. Mansour-Ghanaei F, Fallah MS, Jafarshad R, Joukar F, Salari A, Tavafzadeh R, et al. Prevalence of hepatitis B and hepatitis $\mathrm{C}$, and their risk factors among Guilan blood donors. Sci J Iran Blood Transfus Organ 2008;4:331-6.

57. Mardani A, Ghanbari S, Shahsavarani M, Sahami Zibafar M, Mardani H. Demographic Characteristics of Blood Transmitted Viral Infections in Qom Blood Donors after Bam Earthquake. Iran J Infect Dis Trop Med 2010;15:31-6.

58. Masaeli Z, Jaberi MR, Magsudlu M. A comparison of seroprevalence of blood-borne infections among regular, sporadic, and first-time blood donors in Isfahan. Sci J Iran Blood Transfus Organ 2006;2:301-7.

59. Mirrezaie SM, Saber HR, Hajibeigi B, Salekmoghaddam E, Abbasian A, Alavian SM. Impact of HBV vaccination on prevalence of hepatitis B virus infection among volunteer blood donors in Tehran-Iran. Shiraz E Med $J$ 2014;15:e18066. doi: 10.17795/semj18066.

60. Mohammadali F, Pourfathollah AA. Changes in Frequency of HBV, HCV, HIV and Syphilis Infections among Blood Donors in Tehran Province 2005 - 2011. Arch Iran Med 2014; 17:613-20

61. Mohammadi Tahroodi F, Abdyazdani N, Shakeri F, Rahmani M, Afrazian MS, Abbasi M, et al. The Prevalence of Hep- 
atitis B, Hepatitis C and AIDS in Blood Donors in Ilam Province: A Retrospective Study. Iran J Virol 2018;12:32-9.

62. Mohsenizadeh M, Mollaei HR, Ghaziizadeh M. Seroepidemiological study of hepatitis B, C and HIV among blood donors in Kerman. Asian Pac J Cancer Prev 2017;18:326772. doi: 10.22034/APJCP.2017.18.12.3267.

63. Moniri R, Mosayebii Z, Mossavi GA. Seroprevalence of cytomegalovirus, hepatitis $\mathrm{B}$, hepatitis $\mathrm{C}$ and human immunodeficiency virus antibodies among volunteer blood donors. Iran J Public Health 2004;33:38-42.

64. Nabavizadeh S, Haghbin S. Prevalence of blood transmitted infection in donors of Yasuj blood transfusion organization. J Guilan Uni Med Sci 2000;9:64-7.

65. Niazkar HR, Dorgalaleh A, Rad F. First-time Blood Donors Are Double-edged Swords for Blood Transfusion Centers: A Retrospective Study in Southwest Iran. Turk J Haematol 2020;37:30-5. doi: 10.4274/tjh.galenos.2019.2019.0166.

66. Nour Kojory S, Alaoddowleie H, Seddighian F. Efficacy of confidential self-exclusion and failed systems on blood donation safety in Sari and Behshahr blood donors. Sci J Iran Blood Transfu Organ 2007;4:153-8.

67. OmidKhoda A, Gharehbaghian A, Jamali M, AhmadBeigi N, Hashemi SM, Rahimi A, et al. Comparison of the prevalence of major transfusion-transmitted infections among Iranian blood donors using confidential unit exclusion in an Iranian population: Transfusion-transmitted infections among Iranian blood donors. Hepat Mon 2011;11:11-3.

68. Pourazar A, Akbari N, Hariri M, Yavari F, Akbari S. Evaluation of demographic profiles and prevalence of major viral markers in first time vs repeat blood donors in Esfahan. Sci J Iran Blood Transfu Organ 2006;2:323-9.

69. Ranjbarian P. Comparison of positive HBsAg prevalence in first-time, repeat, and regular blood donors for the purpose of selecting donors in Hamedan Blood Transfusion Center. Sci J Iran Blood Transfus Organ 2008;4:359-63.

70. Rezaie M, Khaleghian A. Prevalence of hepatitis B, hepatitis C and HIV in blood donors in Semnan Province (Iran) from 2011 to 2015. Faslnamahi Kumish 2016;17:501-8.

71. Reza-Zadeh M, Mani Kashani KH, Mohammadi A, Zandevakili H, Lotfi A, Bahrami H, et al. Prevalence of human immunodeficiency, hepatitis B and hepatitis $\mathrm{C}$ viruses in the first time, repeat and regular donors in blood transfusion center, Hamadan, 2004-2005. Iran J Infect Dis Trop Med 2006;11:55-60.

72. Sajjadi SM, Pourfathollah AA, Mohammadi S, Nouri B, Hassanzadeh R, Rad F. The prevalence and trends of hepatitis $\mathrm{B}$, hepatitis $\mathrm{C}$, and HIV among voluntary blood donors in kohgiluyeh and boyer-ahmad transfusion center, Southwestern Iran. Iran J Public Health 2018;47:944-51.

73. Sanei Moghaddam E, Khosravi S, Gharibi T. Prevalence of $\mathrm{HBsAg}$ and Anti-HCV reactivity in donors embarking on direct blood donation and among first-time blood donors in Zahedan Blood Transfusion Center. Sci J Iran Blood Transfu Organ 2005;1:19-25.

74. Seyed Askari SM, Beigzadeh A, Mohammadpoor Ravari
M. The prevalence of transfusion transmitted infections among blood donors in Kerman, Iran. J Kerman Uni Med Sci 2015;22:669-76.

75. Sofian M, Aghakhani A, Izadi N, Banifazl M, Kalan$\operatorname{tar}$ E, Eslamifar A, et al. Lack of occult hepatitis B virus infection among blood donors with isolated hepatitis B core antibody living in an HBV low prevalence region of Iran. Int J Infect Dis 2010;14:e308-10. doi: 10.1016/j. ijid.2009.05.011.

76. Sorouri Zanjani R, Mazloomzadeh S, Koocheki A, Noori M. Prevalence of Hepatitis B, C and HIV Infection in Blood Donors in Zanjan, 2005-2006. Prev Care Nurs Mid $J$ 2013;3:56-63.

77. Taheri Azbarmi z, Nouri S, Joukar F, Jafarshad R, Haajikarimian K, Alinejad s, et al. Transfusion transmitted diseases in Rasht blood donors. Sci J Iran Blood Transfu Organ 2008;4:337-43.

78. Tajbakhsh E, Yaghobi R, Vahedi A. A serological survey on hepatitis $\mathrm{C}$ virus Antibody in blood donors with an ELISA method. Tehran Uni Med J 2007;65:69-73.

79. Vahid T, Alavian SM, Kabir A, Kafaee J, Yektaparast B. Hepatitis B prevalence and risk factors in blood donors in Ghazvin, IR. Iran. Hepat Mon 2005;5:117-22.

80. Vossoughinia H, Taghi Shakeri M, Mokhtari Amirmajdi E, Abedini S. Risk Factors for Hepatitis B and C in 400 Blood Donor Volunteers in Mashhad During 2003-2007: A Casecontrol Study. Horizon Med Sci 2010;15:68-75.

81. Yazhan S, Sohrabi E, Jamili P, Saffari S, Mojaddadi MS. Frequency of HBV, HCV and HIV infections among Sabzevar blood donors based on demographic characteristics during 2009-2013. Sci J Iran Blood Transfus Organ 2016;13:197-206.

82. Zalei B, Pourmand D, Rahmani Y, Bilvaye S. Investigating the prevalence of HTLV and HCV infection in blood donors (for the first time) in blood transfusion organization of Kermanshah, Iran. J Clin Anal Med 2017;8:509-12. doi: 10.4328/JCAM.5540.

83. Hauri AM, Armstrong GL, Hutin YJ. The global burden of disease attributable to contaminated injections given in health care settings. Int J STD AIDS 2004;15:7-16. doi: $10.1258 / 095646204322637182$

84. Jimenez AP, Mohamed MK, Sharaf Eldin N, Abou Seif H, El Aidi S, Sultan Y, et al. Injection drug use is a risk factor for HCV infection in urban Egypt. PloS One 2009;4:e7193. doi: 10.1371/journal.pone.0007193.

85. Candotti D, Laperche S. Hepatitis B Virus Blood Screening: Need for Reappraisal of Blood Safety Measures? Front Med 2018;5:29. doi: 10.3389/fmed.2018.00029.

86. Alavian SM. Hepatitis B virus infection in Iran; Changing the epidemiology. Iran J Clin Infect Dis 2010;5:51-61.

87. Kabir A, Alavian SM, Ahanchi N, Malekzadeh R. Combined passive and active immunoprophylaxis for preventing perinatal transmission of the hepatitis $B$ virus in infants born to HBsAg positive mothers in comparison with vaccine alone. Hepatol Res 2006;36:265-71. doi: 10.1016/j.hepres.2006.08.001. 
88. Mohammadi Z, Keshtkar A, Eghtesad S, Jeddian A, Pourfatholah AA, Maghsudlu M, et al. Epidemiological profile of hepatitis B virus infection in Iran in the past 25 years; a systematic review and meta-analysis of general population studies. Middle East J Dig Dis 2016;8:5-18. doi: 10.15171/ mejdd.2016.01.

89. Schweitzer A, Horn J, Mikolajczyk RT, Krause G, Ott JJ. Estimations of worldwide prevalence of chronic hepatitis $\mathrm{B}$ virus infection: a systematic review of data published between 1965 and 2013. Lancet 2015;386:1546-55. doi: 10.1016/S0140-6736(15)61412-X.

90. Gower E, Estes C, Blach S, Razavi-Shearer K, Razavi H. Global epidemiology and genotype distribution of the hepatitis C virus infection. J Hepatol 2014;61:S45-S57. doi: 10.1016/j.jhep.2014.07.027.

91. Merat S, Rezvan H, Nouraie M, Jafari E, Abolghasemi H, Radmard AR, et al. Seroprevalence of hepatitis C virus: the first population-based study from Iran. Int J Infect Dis 2010;14:113-6. doi: 10.1016/j.ijid.2009.11.032.

92. Alavian SM. Hepatitis C infection in Iran; A review article. Iran J Clin Infect Dis 2009;4:47-59.

93. Nematollahi S, Ayubi E, Almasi-Hashiani A, Mansori K, Moradi Y, Veisani Y, et al. Prevalence of hepatitis C virus infection among high-risk groups in Iran: a systematic review and meta-analysis. Public Health 2018;161:90-8.

94. Almasi-Hashiani A, Ayubi E, Mansori K, Salehi-Vaziri M, Moradi Y, Gholamaliei B, et al. Prevalence of hepatitis B virus infection among Iranian high risk groups: a systematic review and meta-analysis. Gastroenterol Hepatol Bed Bench 2018;11:91-100. 